\title{
Assessment of HIF-1a expression and release following endothelial injury in-vitro and in-vivo
}

\author{
Lamia Heikal ${ }^{1}$, Pietro Ghezzi ${ }^{1}$, Manuela Mengozzi ${ }^{1}$ and Gordon Ferns ${ }^{1,2^{*}}$
}

\begin{abstract}
Background: Endothelial injury is an early and enduring feature of cardiovascular disease. Inflammation and hypoxia may be responsible for this, and are often associated with the up-regulation of several transcriptional factors that include Hypoxia Inducible Factor-1 (HIF-1). Although it has been reported that HIF-1a is detectable in plasma, it is known to be unstable. Our aim was to optimize an assay for HIF-1a to be applied to in vitro and in vivo applications, and to use this assay to assess the release kinetics of HIF-1a following endothelial injury.

Methods: An ELISA for the measurement of HIF-1a in cell-culture medium and plasma was optimized, and the assay was used to determine the best conditions for sample collection and storage. The results of the ELISA were validated using Western blotting and immunohistochemistry $(\mathrm{IHC})$. In vitro, a standardized injury was produced in a monolayer of rat aortic endothelial cells (RAECs) and intracellular HIF-1a was measured at intervals over $24 \mathrm{~h}$. In vivo, a rat angioplasty model was used. The right carotid artery was injured using a $2 \mathrm{~F}$ Fogarty balloon catheter. HIF-1a was measured in the plasma and in the arterial tissue $(0,1,2,3$ and 5 days post injury).

Results: The HIF-1a ELISA had a limit of detection of $2.7 \mathrm{pg} / \mathrm{mL}$ and was linear up to $1000 \mathrm{pg} / \mathrm{mL}$. Between and within-assay, the coefficient of variation values were less than 15\%. HIF-1a was unstable in cell lysates and plasma, and it was necessary to add a protease inhibitor immediately after collection, and to store samples at $-80{ }^{\circ} \mathrm{C}$ prior to analysis. The dynamics of HIF-1a release were different for the in vitro and in vivo models. In vitro, HIF-1a reached maximum concentrations approximately $2 \mathrm{~h}$ post injury, whereas peak values in plasma and tissues occurred approximately 2 days post injury, in the balloon injury model.

Conclusion: HIF-1a can be measured in plasma, but this requires careful sample collection and storage. The carotid artery balloon injury model is associated with the transient release of HIF-1a into the circulation that probably reflects the hypoxia induced in the artery wall.
\end{abstract}

Keywords: HIF-1a, Injury, Endothelial cells, ELISA, Angioplasty

\section{Background}

Endothelial injury is an early and enduring feature of cardiovascular disease. Inflammation and hypoxia may be partially responsible for this, and are often associated with the up regulation of transcriptional factors that include hypoxia-inducible factor-1 (HIF-1) (Ferns and Heikal 2017).

\footnotetext{
* Correspondence: G.Ferns@bsms.ac.uk

${ }^{1}$ Brighton and Sussex Medical School Department of Clinical and experimental investigation, University of Sussex, Falmer East Sussex, Brighton BN1 9PS, UK

${ }^{2}$ Brighton and Sussex Medical School Department of Medical Education, Mayfield House, Falmer East Sussex, Brighton BN1 9PH, UK
}

HIF-1 is a hetero-dimeric transcription factor that consists of an oxygen-regulated $\alpha$ - subunit and a constitutively expressed $\beta$-subunit (Chu and Jones 2016). HIF$1 \beta$ is expressed in the nucleus and its activity is unaffected by hypoxia, whereas the HIF- $1 \alpha$ subunit has a short half-life $(5 \mathrm{~min})$ and its stability is regulated by oxygen tension (Gao et al. 2012). Intracellular HIF-1 $\alpha$ is unstable under normoxic conditions, being rapidly degraded by prolyl hydroxylase enzymes (Semenza 2014). These enzymes are inactivated under low oxygen levels, explaining why HIF-1 $\alpha$ is induced by hypoxia. Some small molecules such as dimethyloxalylglycine (DMOG)

(C) The Author(s). 2018 Open Access This article is distributed under the terms of the Creative Commons Attribution 4.0 International License (http://creativecommons.org/licenses/by/4.0/), which permits unrestricted use, distribution, and 
can stabilize HIF levels under normoxic conditions by inhibiting prolyl hydroxylase (Zhang et al. 2016). Three distinct HIF- $\alpha$ subunits have been identified (HIF-1 $\alpha$, HIF- $2 \alpha$ and HIF- $3 \alpha$ ) that have a tissue-specific pattern of expression (Al Okail 2010). HIF- $1 \alpha$ and HIF $2 \alpha$ are similar but not identical. HIF- $1 \alpha$ is expressed in response to acute hypoxia, whilst HIF- $2 \alpha$ is related to chronic responses (Loboda et al. 2012). HIF-1 drives vascular endothelial growth factor (VEGF) expression and angiogenesis while HIF-2 production blocks angiogenesis by inducing the expression of the soluble VEGF receptor-1, sequestering biologically active, free VEGF. It is possible that HIF- $2 \alpha$ evolved to regulate the VEGF response to hypoxia and the resultant development of the vascular network (Eubank and Marsh 2011; Eubank et al. 2011; Loboda et al. 2012). Therefore, HIF-1 is a key transcription factor in the adaptive responses to low oxygen (Lambert et al. 2010). HIF-1 is involved in the cellular adaptation to injury, inflammation, infection and cancer (Eubank and Marsh 2011). HIF is also induced by inflammatory cytokines including tumour necrosis factor alpha (TNF- $\alpha$ ) and interleukin-1 $\beta$ (IL-1 $\beta)$, that increase the accumulation and transcriptional activity of HIF-1 $\alpha$ (Gao et al. 2012).

HIF-1 also regulates a variety of other important cellular responses affecting a spectrum of protective/reparative processes including angiogenesis, cell proliferation and survival (Ferns and Heikal 2017; Lambert et al. 2010). It promotes the transcription of more than 100 target genes including several angiogenic factors, apart from VEGF, nitric oxide synthase (the inducible and the endothelial forms), platelet-derived growth factor, and erythropoietin (EPO) (Gao et al. 2012; Natarajan et al. 2007).

Several semi-quantitative methods have been used to estimate HIF- $1 \alpha$ levels, for example Western blotting and immunocytochemistry (Karshovska et al. 2007; Srinivasan and Dunn 2011). HIF-1 $\alpha$ levels have also been assessed indirectly by quantifying mRNA expression, or by measuring downstream target genes such as VEGF and EPO (Formento et al. 2005; Park et al. 2014).

Due to the need for a more precise method for HIF- $1 \alpha$ quantification in biological samples, some quantitative methods have also been developed, that include the enzyme linked immunosorbent assay (ELISA) (Formento et al. 2005). Although it has been reported that HIF- $1 \alpha$ is detectable in plasma, (He et al. 2016a) it is unstable and is rapidly degraded under normal oxygen tensions, as discussed above (Park et al. 2014). As a transcription factor, it is normally located within the cell, and there has also been some doubt about its release into plasma (Ferns and Heikal 2017).

Plasma HIF- $1 \alpha$ may potentially represent a biomarker of vascular wall hypoxia, or the extent of atherosclerosis. It may also reflect the severity of hypoxia associated with other pathological conditions including some tumours. Hence, there are a number of possible clinical applications for a plasma HIF-1 $\alpha$ assay.

Our aim was to optimize an assay for HIF- $1 \alpha$ to be applied to in vitro and in vivo studies, and to establish the conditions for sample collection and storage. We then aimed to use the assay to assess the release kinetics of HIF- $1 \alpha$ following endothelial injury in vitro and in vivo.

\section{Methods}

All chemicals were from Sigma Aldrich (Dorset, UK), unless otherwise stated.

\section{Cell culture}

Rat aortic endothelial cells (RAECs) were isolated from the aortae of Sprague Dawley rats (male, 2 weeks old) and used between passages 2-5 (Kobayashi et al. 2005). The cells were cultured in Endothelial cell growth medium supplemented with $10 \%$ fetal bovine serum (FBS) and Penicillin/Streptomycin (final concentration $100 \mathrm{IU} / \mathrm{mL}$ ), and were cultured, prior to our experiments, at $37^{\circ} \mathrm{C}$ in a humidified atmosphere containing $5 \% \mathrm{CO}_{2}$ and $21 \% \mathrm{O}_{2}$. When indicated, experiments under hypoxic conditions were performed under $1 \% \mathrm{O}_{2}, 5 \% \mathrm{CO}_{2}$ and $94 \% \mathrm{~N}_{2}$.

\section{Effect of storage on the HIF-1a stability}

Storage time and temperature, and the effects of adding protease inhibitors to the experimental samples were investigated to determine the optimum storage conditions for HIF- $1 \alpha$ measurements.

For the effect of temperature, aliquots of samples were taken and maintained at different temperatures $(-80$, 20, 4 and $37^{\circ} \mathrm{C}$ ). HIF- $1 \alpha$ concentrations were determined after $24 \mathrm{~h}$ storage using the ELISA as described below.

To determine the effect of storage duration on HIF- $1 \alpha$ concentrations, fresh samples were divided into 3 aliquots, and HIF- $1 \alpha$ was measured immediately, or after 1 and 2 weeks. Aliquots were stored at $-80{ }^{\circ} \mathrm{C}$ until analysis.

To assess the requirement for the addition of a protease inhibitor, aliquots from a set of fresh samples were treated with the protease inhibitor, aprotinin (300 nM) (Mosher et al. 2014). These samples were stored for 1 or 2 weeks at $-80{ }^{\circ} \mathrm{C}$ until they were analyzed.

Linearity, limit of detection and limit of quantification of the assay were determined. Assay precision was assessed by measuring intra and inter-plate replicates with appropriate samples and HIF- $1 \alpha$ standards.

\section{Quantification of HIF-1a after administration of a HIF-1a inducer}

Cells were seeded into 24 well plates with a seeding density of $1 \times 10^{5}$ cell $/ \mathrm{mL}$ and cultured until $80 \%$ confluent. The HIF- $1 \alpha$ inducer; DMOG was added at different concentrations ranging from 0 to $500 \mu \mathrm{M}$ and 
incubated for $24 \mathrm{~h}$ at $21 \% \mathrm{O}_{2}$. Cells incubated in hypoxic conditions $\left(1 \% \mathrm{O}_{2}\right)$ were used as a positive control for HIF- $1 \alpha$ induction. Cells were then lysed and HIF- $1 \alpha$ was measured using the ELISA method. In another set of experiments, cells were similarly treated with different concentrations of DMOG, lysed, and the down-stream effector, VEGF gene expression was measured.

\section{Scratch assay in vitro injury model}

The "scratch assay" is a model of cell/ wound injury, and has been described previously (Heikal et al. 2015). Briefly, a reproducible scratch was produced in the endothelial monolayer, and the cells were then incubated in room air $\left(21 \% \mathrm{O}_{2}\right)$ in a $5 \% \mathrm{CO}_{2}$ incubator. Cells were then lysed and intracellular HIF- $1 \alpha$ assessed. This was evaluated over a period of $24 \mathrm{~h}$ using the ELISA.

\section{Animal studies}

Male Sprague Dawley rats (450 g) were used. All animal experiments were performed under UK Home Office approval according to the Animals Scientific Procedures Act, 1986 and subsequent revisions, and conformed to the Guide for the Care and Use of Laboratory Animals published by the National Institutes of Health (NIH Publication No. 85-23, revised 1996). Studies were designed and conducted in accordance with the ARRIVE guidelines (Kilkenny et al. 2010). Rats were acclimatized in home cages for 1 week prior to experimentation with ad libitum access to food and water and on a $12 \mathrm{~h}$ light-dark cycle.

\section{Quantification of HIF-1a post DMOG administration}

Rats were anesthetized using $2 \%$ isoflurane and placed in a supine position. The right carotid artery was exposed and DMOG was applied locally at different concentrations $(0-7.5 \mathrm{mg} / \mathrm{Kg})$ on the artery using pluronic gel as the vehicle $(30 \% \mathrm{w} / \mathrm{w})$. Three rats were used for each concentration of DMOG. After $24 \mathrm{~h}$, blood was collected from a tail vein, and HIF- $1 \alpha$ was measured by ELISA.

Treated animals were culled after $24 \mathrm{~h}$ and both the right (treated) and left (untreated) carotid arteries were isolated and snap frozen for PCR analysis.

\section{Angioplasty in vivo injury model}

Injury was induced in the rat's carotid artery using a balloon catheter (for angioplasty) as previously described (Tulis 2007). Briefly, rats (450 g) were anesthetized using $2 \%$ isoflurane and placed in a supine position. The right carotid artery was exposed and a 2F Fogarty catheter was used to cause injury. The catheter was inserted into the common carotid artery via the external carotid to cause complete removal of the vascular endothelium from the common carotid artery down to its junction with the aortic arch.

Blood was collected from a tail vein at 0, 1, 2 and 5 days after injury into EDTA containing sample tubes.
Plasma was separated and used for the measurement of HIF- $1 \alpha$ using ELISA.

At the end of the experiment, rats were culled. Both the right (treated) and left (untreated) carotid arteries were isolated and snap frozen for PCR analysis.

In another set of experiments culled animals were perfused fixed with PBS followed by $4 \%$ paraformaldehyde. Both the right (treated) and left (untreated) carotid arteries were isolated and kept in $4 \%$ paraformaldehyde at room temperature for $1 \mathrm{~h}$. Fixed arteries were then placed in a $30 \%$ sucrose solution and left overnight after which they were snap frozen in OCT blocks for cryo-sectioning.

\section{HIF-1a enzyme linked immunosorbent assay (ELISA)}

HIF- $1 \alpha$ was measured using a commercial ELISA kit (R\&D systems/ Biotechne, UK) following the manufacturers' instructions.

a. Standard calibration curves were obtained using HIF-1 $\alpha$ protein standards, allowing the limit of detection, limit of quantification and coefficient of variation to be determined.

b. In vitro samples: Endothelial cells were lysed in $80 \mu \mathrm{L}$ lysis buffer $(25 \mathrm{mmol} / \mathrm{L}$ Tris $\mathrm{HCl} \mathrm{pH} \mathrm{7.} \mathrm{6,}$ $0.1 \%$ SDS, $1 \%$ deoxycholate, $1 \%$ NP40, $0.5 \mathrm{~mol} / \mathrm{L}$ EDTA, $40 \mathrm{mmol} / \mathrm{L}$ EGTA and protease inhibitors). Lysates were then centrifuged at $11000 \mathrm{~g}$ for $15 \mathrm{~min}$ at $4{ }^{\circ} \mathrm{C}$ and the supernatant was collected. Protein concentrations were quantified using a BCA reagent kit (Pierce Biotechnology). Results were expressed as pg/ mg protein.

c. Rat plasma: Blood was collected from the tail vein of each animal and placed into tubes containing EDTA as an anti-coagulant. Blood was kept on ice and centrifuged to separate the plasma, to which a protease inhibitor was immediately added. HIF-1 was then quantified.

\section{Real time qPCR}

The gene expression of vascular endothelial growth factor (VEGF was analysed by quantitative PCR (qPCR). Cells were lysed using TRIzol (Invitrogen, Life Technologies) and RNA was then extracted and purified. RNA quality and concentration were determined using a NanoDrop ND-1000 (NanoDrop Technologies). Reverse transcription and real-time quantitative PCR (qPCR) were carried out on RNA samples for VEGF and $\beta 2$-microglobulin (a housekeeping gene not affected by changes in oxygen levels), using TaqMan gene expression assays (Applied Biosystems/Life Technologies). For gene expression quantification, the comparative threshold cycle $(\Delta \Delta \mathrm{Ct})$ method was used following the manufacturer's instructions. Results were normalized to $\beta 2$-microglobulin expression and 
expressed as arbitrary units using one of the normoxic untreated samples as a calibrator.

For the analysis of rat tissue, RNA was extracted and purified from the frozen artery sections using TRIzol (Invitrogen, Life Technologies). RNA quality and concentrations were determined using a NanoDrop ND1000 (NanoDrop Technologies). Reverse transcription and real-time quantitative PCR (qPCR) were carried out on RNA samples for VEGF and $\beta 2$-microglobulin as described earlier.

\section{Western blotting}

Western blotting was used to verify the presence of HIF$1 \alpha$ in some samples. Cells were lysed and the protein content of the lysate quantified as previously described (Heikal et al. 2015). Thirty $\mu \mathrm{g}$ of cellular proteins were separated on a $10 \%$ SDS-polyacrylamide gel electrophoresis and transferred onto a nitrocellulose membrane (Amersham/ GE Healthcare Life Sciences, Little Chalfont, Buckinghamshire, UK). After blocking with 5\% skimmed milk (for HIF-1 $\alpha$ detection) or $5 \%$ bovine serum albumin; BSA (for GAPDH detection) for $1 \mathrm{~h}$, membranes were incubated with the appropriate primary antibody overnight, followed by HRP-conjugated secondary antibodies for $1 \mathrm{~h}$ at room temperature. HIF- $1 \alpha$ and GAPDH (loading control) were detected using rabbit anti-HIF-1 $\alpha$ (NB100-479, Novus biologicals, UK) and rabbit anti-GAPDH (14C10, Cell Signaling Technology, UK) at 1:500 and 1:1000 respectively and an antirabbit secondary antibody (A0545, Sigma Aldrich, UK) at 1:20,000 dilution. Protein bands were visualized by exposing membranes developed with the ECL reagent (Amersham/ GE Healthcare Life Sciences) to chemiluminescence film (Hyperfilm ECL, Amersham/ GE Healthcare Life Sciences). Bands were quantified using Image J software.

\section{Immunohistochemistry}

Snap frozen carotid arteries were embedded in OCT and were cryo-sectioned (5 $\mu \mathrm{m}$ in thickness) and sections placed onto silane-coated slides. Sections were then washed with PBS and blocked using 10\% donkey serum and $0.3 \%$ Triton $\mathrm{X}-100$ for $1 \mathrm{~h}$ a room temperature, they were incubated with mouse anti-HIF-1 $\alpha$ at 1:50 dilution in $1 \%$ donkey serum at $4{ }^{\circ} \mathrm{C}$ overnight. Donkey antimouse IgG-FITC (sc-2099, Santa Cruz Biotechnology, Germany) (1:100 dilution each) was the secondary antibodies used to detect HIF-1 (green stain). For comparing HIF- $1 \alpha$ expression in different sections, instrument (fluorescence microscope) settings were kept constant for all sections using uninjured arteries as the control. In another set of experiments, cryo-sliced carotid artery sections were used to visualize endothelial cells (ECs) and smooth muscle cells post injury. Arteries were stained with rabbit anti-CD31 (NB100-2284, Novus biologicals, UK) at 1:50 dilution and mouse anti-alpha smooth muscle actin at 1:50 dilution (NB2-33006, Novus biologicals, UK) followed by Alexafluor 647 conjugated anti-rabbit secondary antibody (red stain) and FITC-conjugated anti-mouse secondary antibody (green stain) respectively at 1:200 dilution each. Cellular nuclei were stained with Prolong ${ }^{\oplus}$ Gold anti-fade reagent with DAPI (P36941, Life technologies, UK). Images were obtained using a fluorescence microscope (Leica CTR 5000).

\section{Statistical analysis}

All data were analyzed using Graph Pad Prism 4 software. Differences in treatment were tested for significance using one-way analysis of variance (ANOVA) followed by a Bonferroni correction for multiple comparisons post-hoc test.

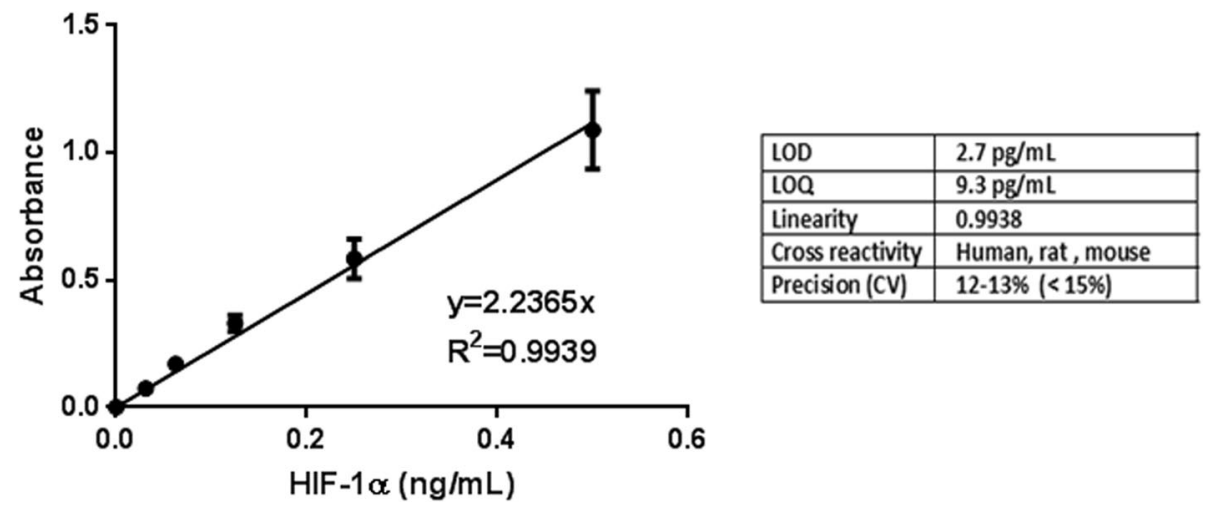

Fig. 1 Calibration curve for standard HIF-1a aprotein at concentration range (0-500 ng/mL) using ELISA assay for protein quantification. The calibration curve was used to determine linearity, the limit of detection and quantification as well as inter and intra variability of the assay. Each point represents the mean \pm SEM of 6 independent experiments 


\section{Results}

Reliability of the ELISA assay for the quantification of HIF$1 a$ in biological samples

Figure 1 shows the results of a typical standard curve using standard recombinant HIF-1 $\alpha$. The assay was linear between 0 and $1000 \mathrm{pg} / \mathrm{mL}(r=0.99)$. The method was sensitive, with a detection limit of $2.7 \mathrm{pg} / \mathrm{mL}$. The ELISA had an intra and inter-assay coefficient of variation of $<15 \%$ and an accuracy of $99.4 \% \pm 8.3 \%$.

The results of the HIF- $1 \alpha$ measurement using the ELISA assay were compared with the findings using the conventional semi-quantitative assay; Western blot. The Western blot in Fig. 2a shows a specific band for the recombinant HIF- $1 \alpha$ standard protein at a molecular weight of approximately130 kDa with a band intensity that was concentration dependent and detectable in the range used (6$200 \mathrm{ng} / \mathrm{mL}$ ). When cell lysates were analyzed by Western blot, we could detect a specific band for HIF-1 $\alpha$ at the molecular weight of $130 \mathrm{kDa}$ in addition to a faint degradation band at $80 \mathrm{kDa}$. (Lidgren et al. 2005; Srinivasan and Dunn 2011) A stronger signal of the higher molecular weight band was detected in cells cultured for 24 in hypoxia. GAPDH was used as a loading control showing a band in each cell lysate at molecular weight $40 \mathrm{kDa}$.

\section{Temperature, storage time and presence of protease} inhibitors are critical for the stability of HIF-1a

Figure 3a shows the detection of HIF- $1 \alpha$ by ELISA in cell lysates kept for $24 \mathrm{~h}$ at different temperatures. Storage at $-80{ }^{\circ} \mathrm{C}$ appears essential for the optimal preservation of the protein. In the experiment shown in Fig. $3 \mathrm{~b}$, samples were kept at $-80{ }^{\circ} \mathrm{C}$ for prolonged periods of time, with and without aprotinin. It is very clear that a protease inhibitor is essential for preventing the degradation of HIF$1 \alpha$ in the stored samples. Other protease inhibitors such as Complete Mini protease inhibitor cocktail (Roche) were tested and showed similar protection against HIF-1 $\alpha$ degradation (data not shown).
Measurement of HIF-1a in cell lysates and plasma samples HIF-1 $\alpha$ was detected in cell lysates treated with DMOG. HIF-1 $\alpha$ was measured in cell lysates using the optimized sample collection discussed in the previous section. HIF-1 $\alpha$ was induced using DMOG. DMOG caused a significant increase HIF-1 $\alpha$ levels when incubated with RAECs for $2 \mathrm{~h}$ at a concentration of $100 \mu \mathrm{M}$ (Fig. 4a) and resulted in transcriptional activation of HIF- $1 \alpha$ target genes, increasing the expression of VEGF gene 3-4 fold (Fig. 4b).

HIF-1a is increased in cell lysate in an in vitro injury model The kinetics of HIF- $1 \alpha$ release was investigated in the scratch assay model. HIF- $1 \alpha$ concentrations in the lysate reached a maximum at approximately $2 \mathrm{~h}$ post injury, after which they gradually returned to baseline (Fig. 5a). This pattern of HIF- $1 \alpha$ levels was mirrored by changes in VEGF gene expression (Fig. 5b).

\section{Circulating HIF-1a in rats treated with DMOG}

HIF- $1 \alpha$ was increased in plasma after treatment of the carotid artery with DMOG applied locally at a concentration ranging from 0 and $7.5 \mathrm{mg} / \mathrm{Kg}$ in a dose-dependent fashion (Fig. 6a). VEGF gene expression in the treated carotid arteries was consistent with the increased levels of plasma HIF-1 $\alpha$ following DMOG administration, but there was no increase in the contralateral, untreated artery (Fig. 6b).

\section{Circulating HIF-1a after in vivo arterial injury}

The kinetics of HIF- $1 \alpha$ release was then measured in a rat model of carotid angiolplasty, using the optimised conditions for plasma sample collection and storage, prior to analysis using the ELISA method. HIF- $1 \alpha$ release reached a maximum at approximately 2 days after injury, and then fell over the next 5 days, but remained above baseline (Fig. 7a). In a separate experiment, we looked at the effect of adding proteinase inhibitors to the blood immediately after collection before plasma

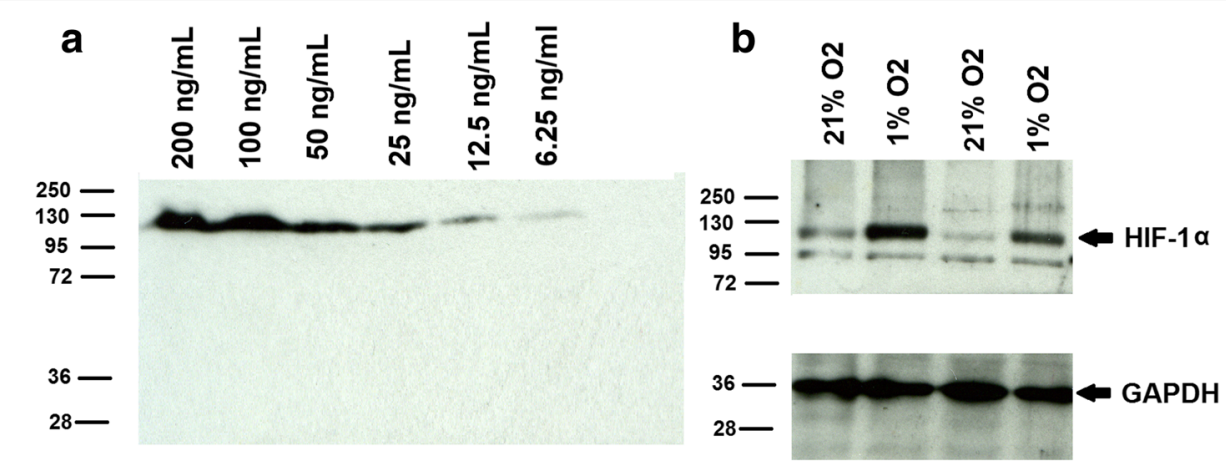

Fig. 2 Western blot analysis of HIF-1a. a Different concentrations of HIF-1a standard protein and $\mathbf{b}$ cell lysates from the cells cultured in $21 \%$ or $1 \% \mathrm{O}_{2}$. HIF-1 a standards and in cell lysates showed a band at a molecular weight of approximately $130 \mathrm{kDa}$. GAPDH (loading control) showed a band at a molecular weight of approximately $36 \mathrm{kDa}$. Numbers on the left indicate the migration of molecular weight standards 

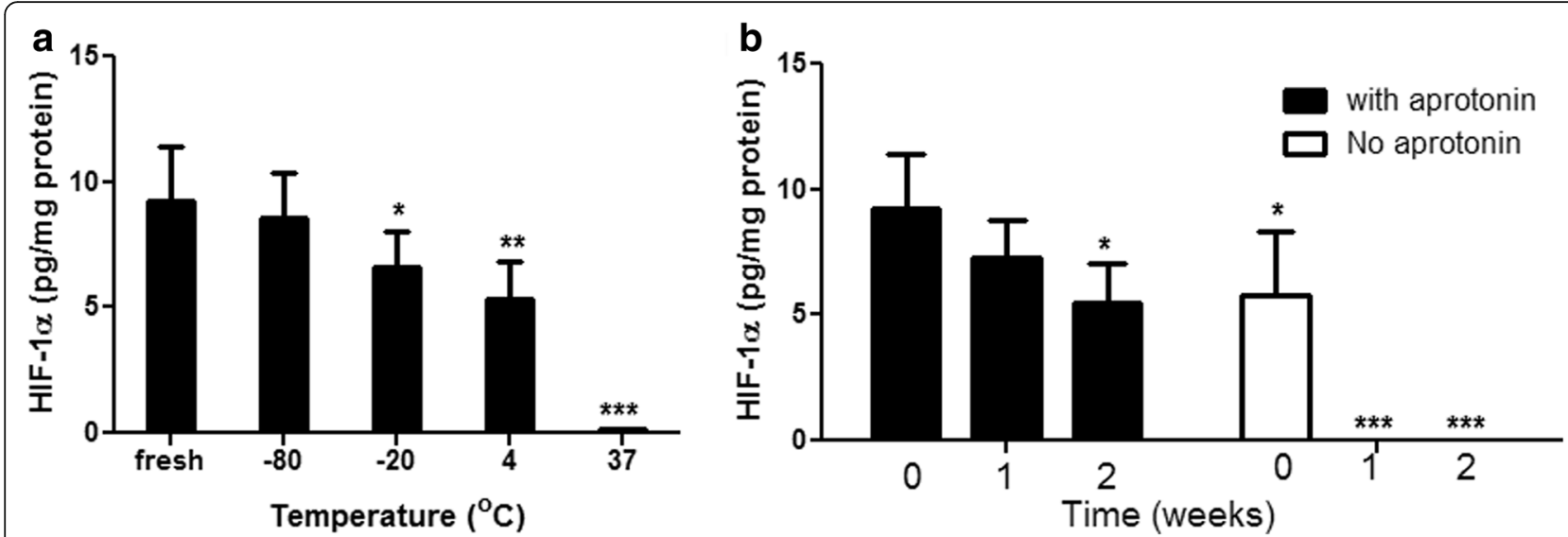

Fig. 3 Effect of (a) temperature, (b) storage time and presence of a protease inhibitor (aprotinin) on the degradation of HIF-1a in cell lysates. The data are mean \pm SEM of 3 independent experiments (triplicates in each experiment), ${ }^{*} P<0.05,{ }^{* *} P<0.01$ and ${ }^{* * *} P<0.001$ compared with freshly collected samples by ANOVA

separation. This has been done by spiking the blood with HIF-1 $\alpha$ protein $(0.6 \mathrm{ng} / \mathrm{mL})$. Blood samples were then split where protease inhibitor was added to one group after which plasma was separated. In the other group, plasma was separated first prior to the addition of protease inhibitor. Samples were then stored for $48 \mathrm{~h}$ prior to analysis. This showed that there was no significant difference between adding the protease inhibitor to the blood immediately after collection or to the plasma after separation and found that this was equally effective. Blood samples were kept on ice after collection and plasma was separated rapidly. Although adding the inhibitor immediately to the blood rather than after plasma separation did not increase the recovery of HIF$1 \alpha$ further (See Additional file 1), this may be more practical in a clinical setting.An increase in local VEGF gene expression in the carotid artery was only observed in injured arteries reaching a maximum level at 2 days after injury, consistent with changes in plasma HIF- $1 \alpha$, and falling to baseline by the fifth day after injury. Uninjured contralateral arteries from the same rats showed no change in VEGF gene expression (Fig. 7b).

\section{HIF-1a localization}

Tissue HIF- $1 \alpha$ levels were also assessed in the injured carotid arteries using immunohistochemistry. An increase was observed in the smooth muscle cells that was maximal 2 days after injury (Fig. 8c) and still detectable at 5 days (Fig. 8d). HIF-1 $\alpha$ was co-localised in the smooth muscle cells after injury.

\section{Discussion}

A reliable and sensitive HIF- $1 \alpha$ ELISA was established and used to assess the stability of HIF- $1 \alpha$ in plasma and
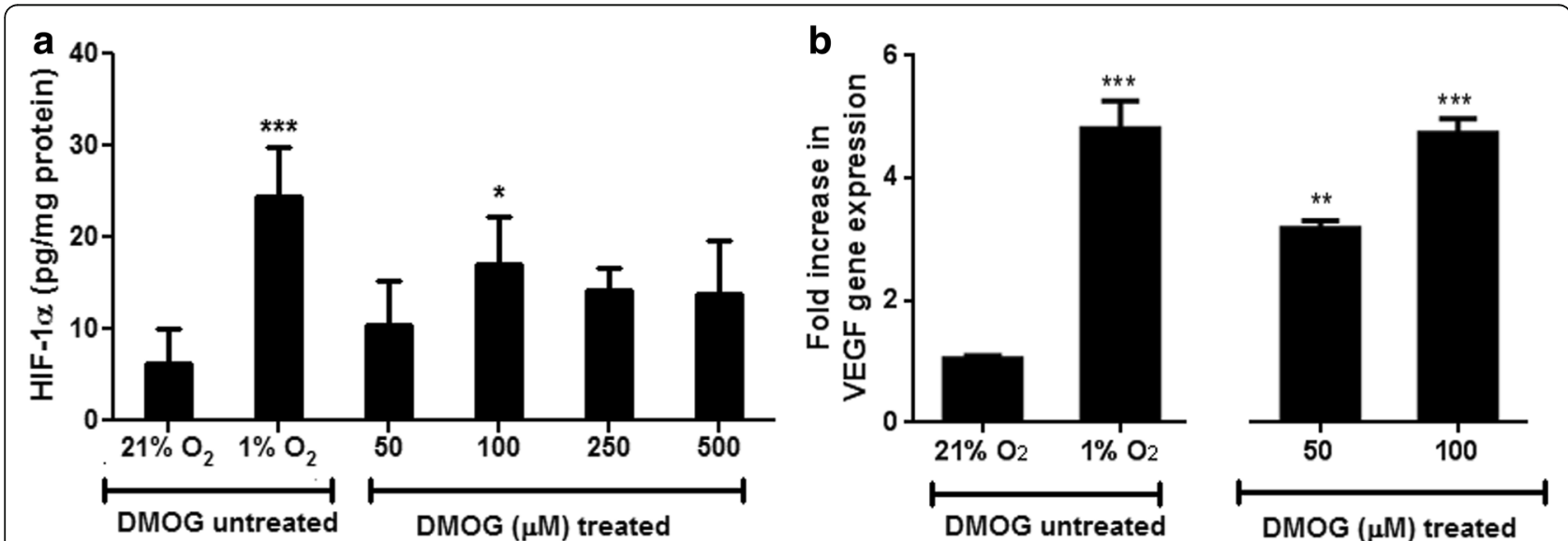

Fig. 4 Concentration response curve showing (a) HIF-1a levels and (b) fold change in VEGF gene expression in cell lysates after treating rat aortic endothelial cells with different concentrations of DMOG (0-500 uM). Cells incubated at 21\% $\mathrm{O}_{2}$ were used as the untreated control group and cells incubated at $1 \% \mathrm{O}_{2}$ were used as a positive control for HIF-1a induction. The data are mean \pm SEM of 6 independent experiments (triplicates in each experiment) where ${ }^{*} P<0.05,{ }^{* *} P<0.01$ and ${ }^{* *} P<0.001$ 



Fig. 5 Time course of the levels of intracellular HIF-1a (a) and VEGF mRNA (b) after a scratch assay in vitro. HIF-1a was measured by ELISA and VEGF mRNA by qPCR. HIF-1a levels are expressed as pg/mg protein and VEGF mRNA levels as arbitrary units versus the uninjured samples. Data are the mean \pm SEM of 6 samples. ${ }^{*} P<0.05, P^{* *}<0.01$ and ${ }^{* * *} P<0.001$

tissue culture medium. Samples could be stored for several days at $-80{ }^{\circ} \mathrm{C}$ but required the addition of aprotinin. HIF- $1 \alpha$ was released following endothelial injury in vitro and in vivo, in the balloon injury model in the rat.

Hypoxia has important effects on intermediary metabolism, cholesterol disposal, and the inflammatory response, and promotes changes in the cellular and extracellular composition of the artery wall that may impact on the response to injury and atherosclerosis. HIF and its down-stream effector gene products have been increasingly recognized for their key role in regulating a wide-spectrum of cellular events, including angiogenesis, cell proliferation, apoptosis and protective response to limit tissue damage (Imtiyaz and Simon 2010; Walshe and D'Amore 2008). There is also increasing evidence that HIF- $1 \alpha$ plays a critical role in an early cardioprotective response (Ke and Costa 2006; Tekin et al. 2010), whilst HIF- $2 \alpha$ mediates a delayed cardio protective effect, playing an important role in adapting the cells to chronic hypoxia and injury (Bautista et al. 2009; Ong and Hausenloy 2012). Therefore measurement of HIF$1 \alpha$ rather than HIF- $2 \alpha$ may be useful for the assessment of acute disease severity and prognosis.

The measurement of HIF-1a in plasma and cells

Although HIF- $1 \alpha$ is known to be unstable, there have been previous reports of the measurement of HIF- $1 \alpha$ in plasma in man and in rats (He et al. 2016b; Li et al. 2015; $\mathrm{Xu}$ et al. 2016). However the description of the sample collection and storage procedures are poorly reported in these studies. In this current paper we have confirmed that HIF- $1 \alpha$ is indeed unstable, and that careful collection procedures are essential to prevent its degradation. We found that blood samples and cell lysates can be stored for up to two weeks at $-80{ }^{\circ} \mathrm{C}$ in the presence of protease inhibitors. Whilst we used aprotinin at a final concentration of $300 \mathrm{nM}$, we found that other protease inhibitors such


Fig. 6 Induction of plasma HIF-1a (a) and VEGF gene expression in the carotid artery tissue (b) after local application of DMOG (0-7.5 mg/Kg) as arbitrary units versus the uninjured samples. Data are the mean $\pm \operatorname{SEM}(n=6),{ }^{*} P<0.05,{ }^{* *} P<0.01,{ }^{* * *} P<0.001$ 

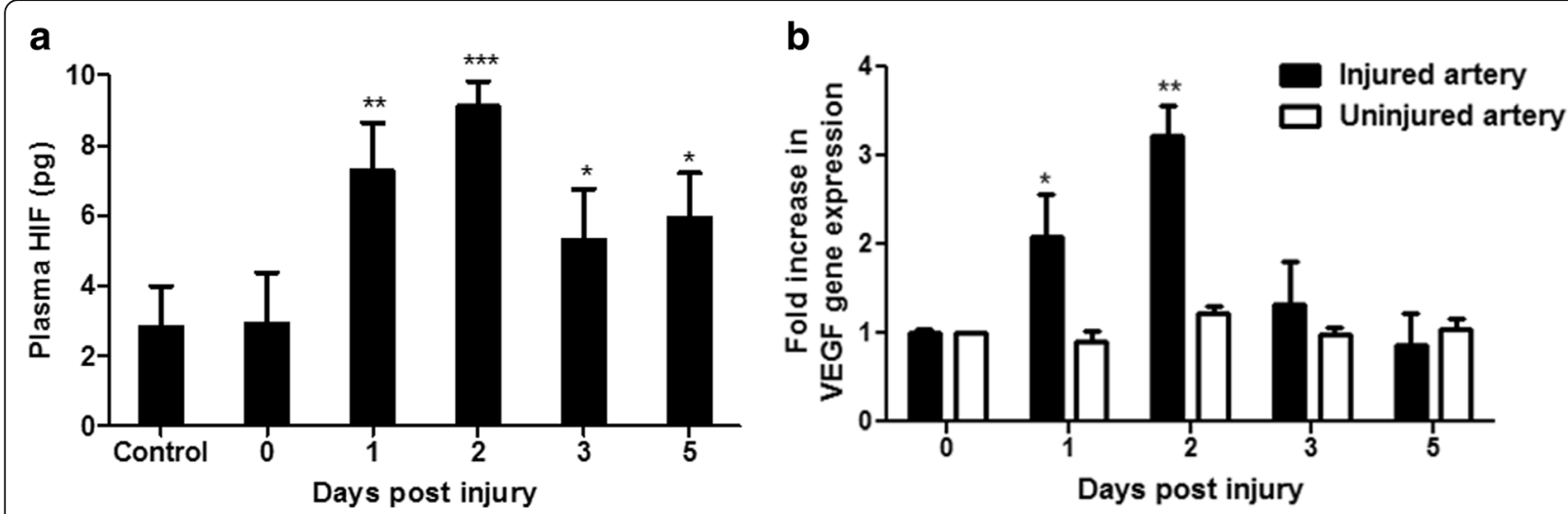

Fig. 7 a Increase in plasma HIF-1a in a rat angioplasty model. Plasma levels of HIF-1a, measured by ELISA, increased after injury, reaching a maximum after $48 \mathrm{~h}$. (b) VEGF mRNA levels in the injured carotid and the contralateral carotid of the same rats as arbitrary units versus the uninjured samples. Data are mean $\pm \operatorname{SEM}(n=6) .{ }^{*} P<0.05,{ }^{* *} P<0.01$ and ${ }^{* * *} P<0.001$

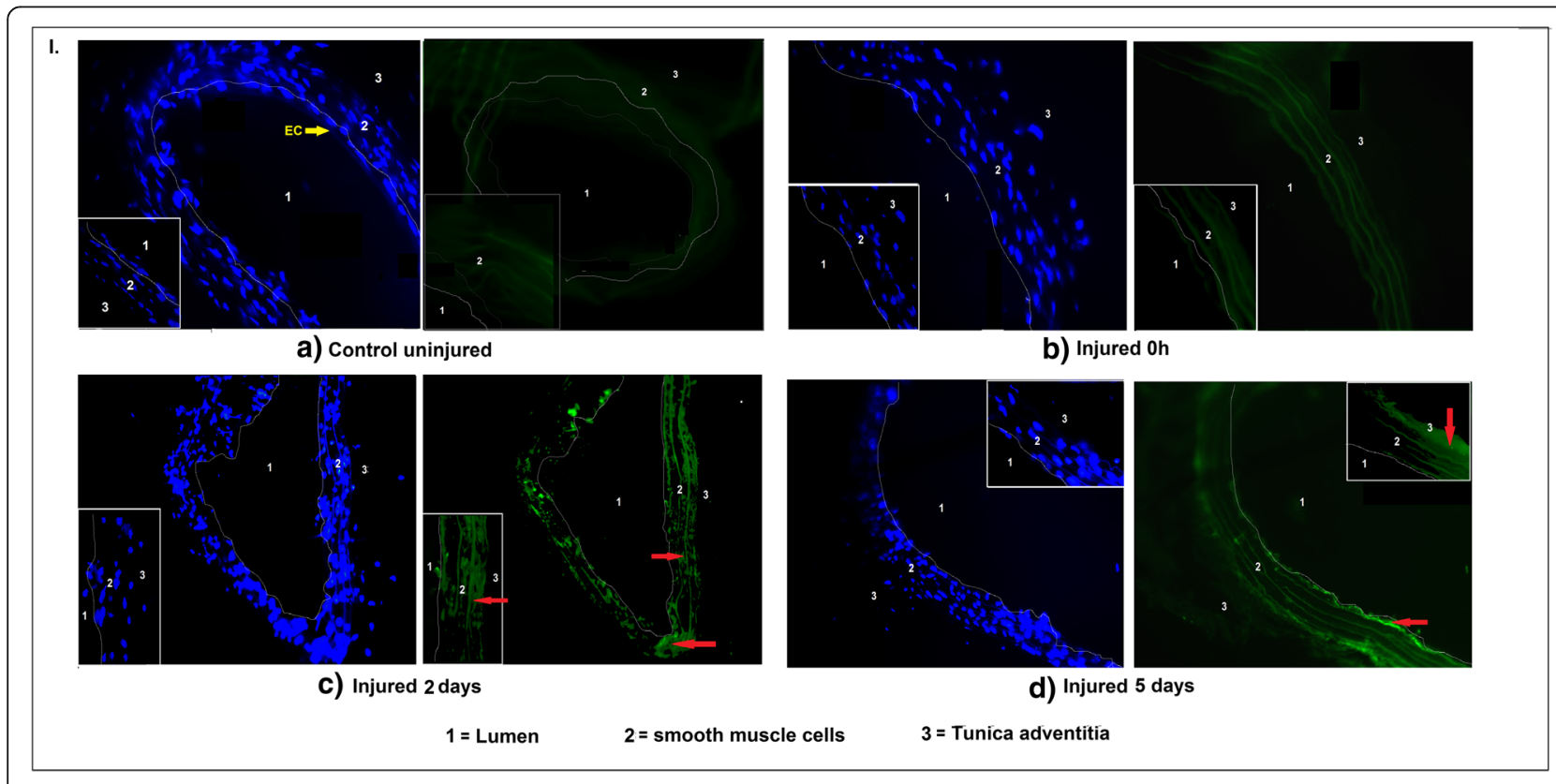

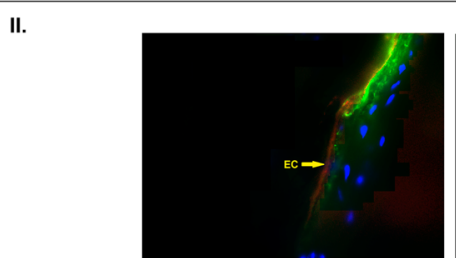

a)Control uninjured

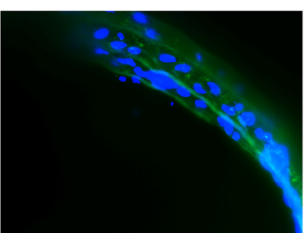

b) Injured Oh

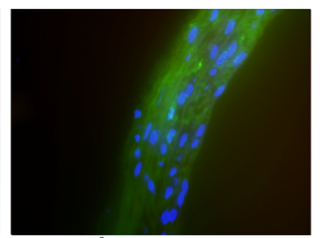

C) Injured 2 days

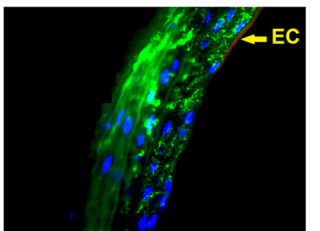

d) Injured 5 days

Fig. 8 Panel I represents immunohistochemistry of (a) untreated carotid arteries and injured carotid arteries (b) immediately after injury (c) 2 days post injury and (d) 5 days post injury using a Leica CTR 5000 fluorescence microscope. Arteries were stained with mouse anti-HIF-1a and followed by FITC-conjugated anti-mouse secondary antibody (green stain) to visualize HIF-1a. Nuclei were stained with DAPI (Blue stain). 1 represents the lumen, 2 represents the smooth muscle cells and 3 represents the Tunica adventitia. Images shown are at 40x magnification and 63x magnification (inset images). Red arrows indicate the localization of HIF-1a expression in the smooth muscle cells. Panel II represents the staining of endothelial cells (EC) and smooth muscle cells (SMCs). The yellow arrow indicates EC layer in control (uninjured) arteries which is removed post injury and starts to regenerate 5 days post injury. Arteries were stained with rabbit anti-CD31 and mouse anti-alpha smooth muscle actin followed by Alexafluor 647 conjugated anti-rabbit secondary antibody (red stain) and FITC-conjugated anti-mouse secondary antibody (green stain) to visualize EC and SMCs respectively 
as Complete Mini protease inhibitor cocktail tablets (Roche) have a similar effect (data not shown).

The HIF-1 $\alpha$ ELISA was robust, of adequate sensitivity and precision for use in biological samples. The values obtained using the ELISA were consistent with Western blot analysis using different HIF- $1 \alpha$ antibodies. The measurement of HIF- $1 \alpha$ in the in vitro studies was also consistent with expectations: hypoxia and a known HIF-1 $\alpha$ inducer increased intracellular HIF- $1 \alpha$ levels and down-stream VEGF expression. HIF- $1 \alpha$ is involved in regulating the expression of VEGF, which is one of the most potent proangiogenic growth factors (Song et al. 2017). The expression of VEGF was measured to confirm that the levels of HIF- $1 \alpha$ in the biological samples were sufficient to cause down-stream signalling.

We observed that there was a transient increase in the expression of HIF-1 $\alpha$ and VEGF in a model of rat aortic endothelial cell (RAECs) injury in vitro. The rapid increase in HIF-1 $\alpha$ levels from injured RAECs reached a peak level at approximately $2 \mathrm{~h}$ post injury in the scratch model. This occurred under normoxic conditions and in the absence of inflammatory cells showing that HIF- $1 \alpha$ is directly involved in the adaptive response to cell injury.

\section{HIF-1a and vascular injury}

vIn vivo, DMOG, applied locally to a rat carotid artery, caused a systemic increase in plasma HIF-1 $\alpha$ that peaked at 2 days, and was approximately 3 -fold higher than baseline values. This was associated with an upregulation of the local, but not the contralateral VEGF arterial tissue expression. HIF-1 $\alpha$ appears to be an important mediator of the pro-atherogenic cellular response to hypoxia and is up-regulated in inflammatory and hypoxic areas of carotid arteries in simple and complex lesions from patients with carotid artery disease. In vascular smooth muscle cells, HIF-1 $\alpha$ can be activated under normoxic conditions by platelet products such as platelet derived growth factor and thrombin (Gorlach et al. 2001). In the rat carotid balloon injury model, platelets are deposited on the injured artery within minutes of injury, and that they are important in the subsequent formation of the neo-intima (Fingerle et al. 1989). In a murine model of vascular injury, HIF- $1 \alpha$ expression was shown to be increased in the smooth muscle cells of the tunica media, as early as 1 day after injury, HIF- $1 \alpha$ mRNA expression was induced at $6 \mathrm{~h}$ after injury, and its inhibition by the local application of HIF- $1 \alpha-$ siRNA reduced neo-intimal area by $49 \%$ and significantly decreased the neo-intimal smooth muscle cells content. HIF- $1 \alpha$ expression therefore appears to be directly involved in the formation of the neo-intima after vascular injury (Karshovska et al. 2007).

\section{Conclusions}

It is possible to measure HIF- $1 \alpha$ in biological samples using an ELISA assay, although careful sample preparation and storage is essential. This may be of value in conditions in which tissue hypoxia may play a role, including cancer, cardiovascular disease and pulmonary disease.

HIF- $1 \alpha$ is rapidly up-regulated in an in vitro, woundhealing model, the scratch assay, even in the absence of hypoxia. It is therefore likely to play an important role in orchestrating the response to injury.

The up-regulation of HIF- $1 \alpha$ expression and release was more sustained in the rat balloon injury model, where plasma HIF-1 $\alpha$ reached its peak concentrations 2 days post injury. The differences in the kinetics of HIF- $1 \alpha$ expression and release for the in vitro and in vivo models could be due to the complexity of the in vivo model. The latter model involves several cell types, including VSMS, platelets and inflammatory cells; these may interact to stimulate pathways that could lead to a more sustained HIF release that may have implications for conditions such as coronary atherosclerosis, restenosis and stroke.

\section{Additional file}

Additional file 1: Figure S1. Effect of adding the protease inhibitor; aprotinin on HIF-1a levels in plasma when added directly to the blood after collection versus addition to the plasma after separation. Blood was spiked with HIF-1a protein $(0.6 \mathrm{ng} / \mathrm{mL})$. Blood samples were then split into two groups where the protease inhibitor was added to one group after which plasma was separated. In the other group, plasma was separated first prior to the addition of protease inhibitor. Data represented are the mean \pm SEM of 3 rats (triplicate of each). (DOCX $23 \mathrm{~kb})$

\section{Abbreviations}

DMOG: Dimethyloxalylglycine; ELISA: Enzyme linked immunosorbent assay; HIF-1a: Hypoxia inducible factor-1 alpha; RAECs: Rat aortic endothelial cells; VEGF: Vascular endothelial growth factor

\section{Acknowledgements}

I would like to acknowledge Willie-Henri Quah, a Junior research associate medical students who did some of the preliminary work for this study.

\section{Funding}

This work has been funded by Brighton and Sussex Medical School.

\section{Availability of data and materials}

The datasets used and/or analyzed during the current study are available from the corresponding author on reasonable request. All data generated or analyzed during this study are included in this published article.

\section{Authors' contributions}

GF, LH and PG designed the research study. LH performed the research. MM provided help and advice on the ELISA experiments. LH analyzed the data. LH, GF and PG wrote the manuscript. All authors contributed to editorial changes in the manuscript. All authors read and approved the final manuscript.

\section{Ethics approval}

All animal experiments were performed under UK Home Office approval according to the Animals Scientific Procedures Act, 1986. All the work was done under the Home-office personal and project license (PIL 70/23855, PPL 70/7893). 


\section{Competing interests}

The authors declare they have no competing interests as defined by Molecular Medicine, or other interests that might be perceived to influence the results and discussion reported in this paper.

\section{Publisher's Note}

Springer Nature remains neutral with regard to jurisdictional claims in published maps and institutional affiliations.

Received: 28 March 2018 Accepted: 7 May 2018

\section{Published online: 16 May 2018}

\section{References}

Al Okail MS. Cobalt chloride, a chemical inducer of hypoxia-inducible factor-1 alpha in U251 human glioblastoma cell line. Journal of Saudi Chemical Society. 2010;14:197-201. https://doi.org/10.1016/j.jscs.2010.02.005.

Bautista L, Castro MJ, López-Barneo J, Castellano A. Hypoxia inducible factor-2a stabilization and maxi-K+ channel $\beta 1$-subunit gene repression by hypoxia in cardiac myocytes. Role in preconditioning. Circ Res. 2009;104:1364-72. https://doi.org/10.1161/circresaha.108.190645.

Chu HX, Jones NM. Changes in hypoxia-inducible Factor-1 (HIF-1) and regulatory prolyl hydroxylase (PHD) enzymes following hypoxic-ischemic injury in the neonatal rat. Neurochem Res. 2016;41:515-22. https://doi.org/10.1007/ s11064-015-1641-y.

Eubank TD, Marsh CB. HIFs: a-cute answer to inflammation? Blood. 2011;118:4857. https://doi.org/10.1182/blood-2011-05-355164.

Eubank TD, Roda JM, Liu HW, O'Neil T, Marsh CB. Opposing roles for HIF-1 alpha and HIF-2 alpha in the regulation of angiogenesis by mononuclear phagocytes. Blood. 2011;117:323-32. https://doi.org/10.1182/blood-2010-01261792.

Ferns GAA, Heikal L. Hypoxia in Atherogenesis. Angiology. 2017;68:472-93. https://doi.org/10.1177/0003319716662423.

Fingerle J, Johnson $\mathrm{R}$, Clowes AW, Majesky MW, Reidy MA. Role of platelets in smooth muscle cell proliferation and migration after vascular injury in rat carotid artery. Proc Natl Acad Sci U S A. 1989;86:8412-6. https://doi.org/10. 1073/pnas.86.21.8412.

Formento JL, Berra E, Ferrua B, Magné N, Simos G, Brahimi-Horn C, Pouysségur J, Milano G. Enzyme-Linked Immunosorbent Assay for Pharmacological Studies Targeting Hypoxia-Inducible Factor 1a. Clinical and Diagnostic Laboratory Immunology. 2005;12:660-4. https://doi.org/10.1128/CDLI.12.5.660-664.2005.

Gao LG, Chen Q, Zhou XL, Fan L. The role of hypoxia-inducible factor 1 in atherosclerosis. J Clin Pathol. 2012;65:872-6. https://doi.org/10.1136/jclinpath2012-200828

Gorlach A, Diebold I, Schini-Kerth VB, Berchner-Pfannschmidt U, Roth U, Brandes RP, Kietzmann T, Busse R. Thrombin activates the hypoxia-inducible factor-1 signaling pathway in vascular smooth muscle cells role of the p22(phox)containing NADPH oxidase. Circ Res. 2001;89:47-54. https://doi.org/10.1161/ hh1301.092678.

He J, Hu Y, Hu M, Zhang S, Li B. The relationship between the preoperative plasma level of HIF-1a and clinic pathological features, prognosis in nonsmall cell lung cancer. Sci Rep. 2016a;6:20586. https://doi.org/10.1038/ srep20586.

He JB, Hu Y, Hu MM, Zhang SY, Li BL. The relationship between the preoperative plasma level of HIF-1 alpha and clinic pathological features, prognosis in non-small cell lung cancer. Sci Rep. 2016b;6 https://doi.org/10.1038/ srep20586.

Heikal L, Ghezzi P, Mengozzi M, Ferns G. Low oxygen tension primes aortic endothelial cells to the reparative effect of tissue-protective cytokines. Mol Med. 2015;21:709-16.

Imtiyaz HZ, Simon MC. Hypoxia-inducible factors as essential regulators of inflammation. Curr Top Microbiol Immunol. 2010;345:105-20. https://doi.org/ 10.1007/82_2010_74.

Karshovska E, Zernecke A, Sevilmis G, Millet A, Hristov M, Cohen CD, Schmid H, Krotz F, Sohn HY, Klauss V, et al. Expression of HIF-1 alpha in injured arteries controls SDF-1 alpha-mediated neointima formation in apolipoprotein Edeficient mice. Arteriosclerosis Thrombosis and Vascular Biology. 2007:27: 2540-7. https://doi.org/10.1161/atvbaha.107.151050.

Ke OD. Costa M. Hypoxia-inducible factor-1 (HIF-1). Mol Pharmacol. 2006;70:1469_ 80. https://doi.org/10.1124/mol.106.027029
Kilkenny C, Browne WJ, Cuthill IC, Emerson M, Altman DG. Improving bioscience research reporting: the ARRIVE guidelines for reporting animal research. PLOS Biol. 2010;8:e1000412. https://doi.org/10.1371/journal.pbio.1000412.

Kobayashi M, Inoue K, Warabi E, Minami T, Kodama T. A simple method of isolating mouse aortic endothelial cells. J Atheroscler Thromb. 2005;12:13842. https://doi.org/10.5551/jat.12.138.

Lambert CM, Roy M, Robitaille GA, Richard DE, Bonnet S. HIF-1 inhibition decreases systemic vascular remodelling diseases by promoting apoptosis through a hexokinase 2-dependent mechanism. Cardiovasc Res. 2010;88:196204. https://doi.org/10.1093/cvr/cvq152.

Li WL, Wang KJ, Liu ZW, Ding WG. HIF-1 alpha change in serum and callus during fracture healing in ovariectomized mice. Int J Clin Exp Pathol. 2015;8:117-26.

Lidgren A, Hedberg Y, Grankvist K, Rasmuson T, Vasko J, Ljungberg B. The expression of hypoxia-inducible factor $1 \mathrm{a}$ is a favorable independent prognostic factor in renal cell carcinoma. Clin Cancer Res. 2005;11:1129-35.

Loboda A, Jozkowicz A, Dulak J. HIF-1 versus HIF-2 - is one more important than the other? Vasc Pharmacol. 2012;56:245-51. https://doi.org/10.1016/j.vph. 2012.02.006.

Mosher RA, Coetzee JF, Allen PS, Havel JA, Griffith GR, Wang C. Effects of sample handling methods on substance $P$ concentrations and immunoreactivity in bovine blood samples. Am J Vet Res. 2014;75:109-16. https://doi.org/10.2460/ ajvr.75.2.109.

Natarajan R, Salloum FN, Fisher BJ, Ownby ED, Kukreja RC, Fowler AA. Activation of hypoxia-inducible factor-1 via prolyl-4 hydoxylase-2 gene silencing attenuates acute inflammatory responses in postischemic myocardium. Am J Phys Heart Circ Phys. 2007;293:H1571-80. https://doi.org/10.1152/ajpheart. 00291.2007.

Ong S-G, Hausenloy DJ. Hypoxia-inducible factor as a therapeutic target for cardioprotection. Pharmacol Ther. 2012;136:69-81. https://doi.org/10.1016/j. pharmthera.2012.07.005.

Park SR, Kinders RJ, Khin S, Hollingshead M, Antony S, Parchment RE, Tomaszewski JE, Kummar S, Doroshow JH. Validation of a hypoxia-inducible factor-1a specimen collection procedure and quantitative ELISA in solid tumor tissues. Anal Biochem. 2014;459:1-11. https://doi.org/10.1016/j.ab.2014.04.025.

Semenza GL. Hypoxia-inducible factor 1 and cardiovascular disease. In: Julius D, editor. Annual review of physiology, vol. 76; 2014. p. 39-56.

Song SL, Xiao XY, Guo D, Mo LQ, Bu C, Ye WB, Den OW, Liu ST, Yang XX. Protective effects of Paeoniflorin against AOPP-induced oxidative injury in HUVECS by blocking the ROS-HIF-1 alpha/NEGF pathway. Phytomedicine. 2017;34:115-26. https://doi.org/10.1016/j.phymed.2017.08.010.

Srinivasan S, Dunn JF. Stabilization of hypoxia-inducible factor-1a in buffer containing cobalt chloride for western blot analysis. Anal Biochem. 2011;416: 120-2. https://doi.org/10.1016/j.ab.2011.04.037.

Tekin D, Dursun AD, Xi L. Hypoxia inducible factor 1 (HIF-1) and cardioprotection. Acta Pharmacol Sin. 2010;31:1085-94. https://doi.org/10.1038/aps.2010.132.

Tulis DA. Rat carotid artery balloon injury model. Methods in molecular medicine. 2007;139:1

Walshe TE, D'Amore PA. The role of hypoxia in vascular injury and repair. Annual Review of Pathology: Mechanisms of Disease. 2008;3:615-43. https://doi.org/ 10.1146/annurev.pathmechdis.3.121806.151501.

Xu M, Zhang Y, Tang L, Huang H. Concentration analysis of hypoxia-inducible factor-1 alpha and vascular endothelial growth factor in patients with aortic aneurysm at different stages and its clinical significance. Cell Mol Biol. 2016; 62:73-6.

Zhang S, Ma K, Liu YW, Pan XB, Chen QM, Qi L, Li SJ. Stabilization of hypoxiainducible factor by DMOG inhibits development of chronic hypoxia-induced right ventricular remodeling. J Cardiovasc Pharmacol. 2016;67:68-75. https:// doi.org/10.1097/fjc.0000000000000315. 